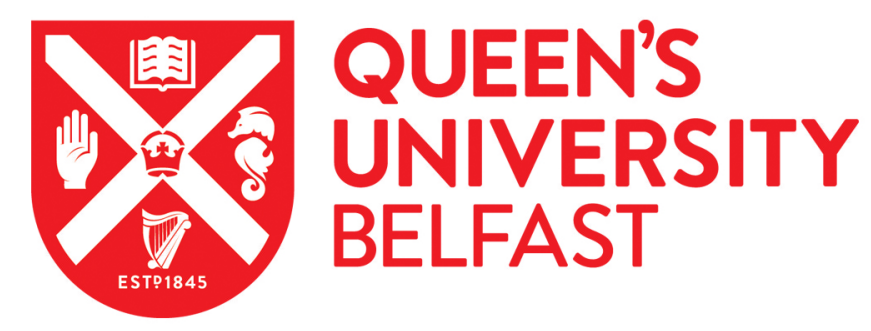

\title{
Lap Time Simulation Tool for the Development of an Electric Formula Student Car
}

Doyle, D., Cunningham, G., White, G., \& Early, J. (2019). Lap Time Simulation Tool for the Development of an Electric Formula Student Car. In WCX SAE World Congress Experience 2019: Proceedings (SAE Technical Papers). SAE International. https://doi.org/10.4271/2019-01-0163

Published in:

WCX SAE World Congress Experience 2019: Proceedings

Document Version:

Peer reviewed version

Queen's University Belfast - Research Portal:

Link to publication record in Queen's University Belfast Research Portal

Publisher rights

Copyright 2019 SAE.

This work is made available online in accordance with the publisher's policies. Please refer to any applicable terms of use of the publisher.

\section{General rights}

Copyright for the publications made accessible via the Queen's University Belfast Research Portal is retained by the author(s) and / or other copyright owners and it is a condition of accessing these publications that users recognise and abide by the legal requirements associated with these rights.

Take down policy

The Research Portal is Queen's institutional repository that provides access to Queen's research output. Every effort has been made to ensure that content in the Research Portal does not infringe any person's rights, or applicable UK laws. If you discover content in the Research Portal that you believe breaches copyright or violates any law, please contact openaccess@qub.ac.uk. 


\section{Lap Time Simulation Tool for the Development of an Electric Formula Student Car Author, co-author (Do NOT enter this information. It will be pulled from participant tab in MyTechZone) Affiliation (Do NOT enter this information. It will be pulled from participant tab in MyTechZone)}

\begin{abstract}
This work details the development of a lap time simulation (LTS) tool for use by Queen's University Belfast in the Formula Student UK competition. The tool provides an adaptable, user-friendly virtual test environment for the development of the team's first electric vehicle. A vehicle model was created within Simulink, and a series of events simulated to generate the performance envelope of the car in the form of maximum combined lateral/longitudinal accelerations against velocity (ggv diagram). A four-wheeled vehicle including load transfer was modelled, capturing shifts in traction between each tire, which can influence performance in vehicles where the total tractive power is split between individual wheel motors. The acceleration limits in the ggv diagram were used to simulate the acceleration and endurance events at Formula Student. These events were simulated using a MATLAB code considering a point mass, quasi-steady state model with a perfect driver. This method considering all four wheels captures performance characteristics that point mass models normally cannot, without the complexity and time required for more detailed LTS solutions including yaw movements and driver models. It also separates the vehicle model from the MATLAB code required to run the LTS, reducing the complexity of implementing future changes. The LTS was benchmarked against the freeware tool OptimumLap and validated where possible against competition results. A Latin Hypercube sampling technique was employed to generate numerous input scenarios for the simulation, and a response surface fitted to the results to perform a sensitivity analysis. Results from this analysis indicate several areas to efficiently focus future resource allocation as well as attempting to quantify trade-offs. Optimum powertrain gear ratio and battery capacity for a proposed vehicle were specified using the tool.
\end{abstract}

\section{Introduction}

Formula Student (FS) is an annual competition which "challenges students to conceive, design, fabricate, and compete with small formula-style racing cars" [1]. With the competition occurring every year, teams face issues in the form of rapid development and limited testing time, as well as knowledge loss between subsequent years as team members leave their university. Teams compete in various events, the most critical of which for the purposes of this work being the $75 \mathrm{~m}$ acceleration event and the $22 \mathrm{~km}$ endurance event.

Alongside the rise in popularity of passenger electric vehicles (EVs), FS has seen an increasing number of EVs competing against internal combustion engine (ICE) cars each year since their introduction to the competition in 2012. Queen's University Belfast (QUB) aim to compete in the competition with an EV for the first time in 2020 with the proposed car featuring four wheel drive in the form of four individually powered in-hub motors with speed reduction gearing.
Variation of many vehicle design parameters rarely yield a linear or easily predictable change in performance, with many changes involving trade-offs which must be evaluated. Real world testing is both expensive and time consuming, and evaluation of new concepts may require the production of several prototypes. Simulation through a computer-based vehicle model provides a virtual test environment, allowing the evaluation of new concepts or design decisions to be done quicker and at a much lower cost. A method of LTS is particularly useful to QUB due to the short development time available each year for FS and the increased ability to influence design decisions in the current early stages of development.

\section{Background}

Simulation is widely carried out within FS, professional racing and the commercial automotive industry with varying degrees of complexity. Driver-in-the-loop simulators have a human driver provide steering and pedal inputs in order to navigate a track, who may in turn receive motion feedback and immersive surrounding graphics. The underlying vehicle models are generally very detailed, with tire models obtained from test data, yaw motion, transient suspension effects and multibody dynamics. These are widely adopted in Formula One, with the added benefit of driver training as well as virtual testing - however testing or comparison between vehicle models is subject to human variability.

Replacing the human driver with a controller (driver model) allows LTS to be carried out automatically and consistently, albeit with additional challenges. The commercial automotive or transport industries make use of standard drive cycles and program the driver model to follow a specified velocity profile, using bespoke models or flexible packages such as AVL CRUISE (as in [2]). This is obviously less useful in LTS for racing where the goal is to maximize the velocity profile around a track of specified geometry. Non-linear or discontinuous vehicle performance (for example when maximum velocity is reached) must be accounted for within the driver model. Packages such as IPG CarMaker have been used for FS applications [3], which are again generally complex and aimed towards the detailed design stages.

A simpler method is to model the vehicle as a point mass, single-tire vehicle, with a constant coefficient of friction tire model. This removes the necessity of detailed tire models, and reduces complexity by negating yaw motion - the point mass vehicle can neither oversteer nor understeer and navigates along a specified course only under the action of combined longitudinal and lateral tire forces. Using the limits of performance (either traction limited or power limited) to produce the lateral and longitudinal accelerations represents a perfect driver, which although non-realistic is consistent 
for each simulation. The assumptions and simplifications employed with this method are at the expense of accuracy, although provide a comparative estimate between different setups and can be used without needing comprehensive information or data from the vehicle.

OptimumLap is a free LTS tool featuring a point mass, quasi-steady state model, used by Centro Universitário FEI in [4]. OptimumLap quote an accuracy of within $10 \%$ in their documentation [5], showing reasonable accuracy for a simple model. However, single tire, point mass models cannot simulate some characteristics important to the specification of powertrains with four independently powered wheels, as proposed by QUB. Under acceleration, load transfer causes the normal force at each tire to change, therefore changing the available traction. Consider the case where a vehicle moving at an instantaneous velocity has a total available traction power of $100 \mathrm{~kW}$ and four in-hub motors capable of $30 \mathrm{~kW}$ each. Under a single tire model, there is more motor power available than traction power, and as such $100 \mathrm{~kW}$ is transmitted to the road. Now consider $70 \%$ of the load shifts to the rear under acceleration. Now, $70 \mathrm{~kW}$ of traction is available at the rear, with only $60 \mathrm{~kW}$ of motor power available. Thus for the same available traction and available motor power, $10 \mathrm{~kW}$ less power is transmitted to the road.

Sakhalkar et al. avoid this by using a longitudinal vehicle model which includes longitudinal load transfer under acceleration [6]. The vehicle treats corners as an equivalent straight section with a velocity restriction and thus reduces the track to a longitudinal problem.

However this doesn't account for the variance in traction between the left and right tires through lateral load transfer. Additionally, only constant speed, steady-state cornering is modelled, compared to the quasi-steady combined cornering and acceleration/braking allowed within OptimumLap. The maximum cornering velocity is a function of only the tire coefficient of friction and the local track radius. This does not factor in downforce or the longitudinal tire force required to maintain constant velocity, which along with quasi-steady state cornering are considered necessary in a robust LTS tool for QUB.

\section{Scope}

The goal of this work was to produce an LTS tool to aid in the development of the current proposed and future QUB EVs for FS. The tool had the following aims:

1. Specify optimum transmission ratio and battery pack capacity.

2. Guide future EV development and funding allocation through a multivariate sensitivity analysis.

3. Completion within the 24 week university calendar.

4. Address the issue of annual knowledge loss through a model which is easily adaptable to future design changes and requires little understanding of the underlying methodology to use.

This paper details the methodology behind an LTS tool developed within MATLAB and Simulink which achieves the listed aims. Due to the combination of a short time frame and an absence of available tire and vehicle data, the development of the LTS tool was constrained to a point mass quasi-steady state vehicle model. A method of addressing point mass models' inability to capture variations in traction under load transfer is implemented and discussed. OptimumLap was chosen for comparison as it represents a baseline point mass model without the additional methodology described in this paper, has proven accuracy and is used and encouraged within FS. Additionally it is more focused towards the early concept design stages than more advanced packages.
Powertrain components are sized using the developed LTS tool and a sensitivity analysis is performed.

\section{Methodology}

A longitudinal vehicle model was created within Simulink, including four separate drivetrains to represent each motor and their mechanical connection to the wheels. Each motor is capable of delivering different amounts of torque depending on the instantaneous traction available at the wheel, whilst staying within the $80 \mathrm{~kW}$ total power limit that applies to EVs in the competition [7].

A simple constant friction coefficient tire model was used in the absence of tire test data, with the maximum tire tractive force in the form of Eq. (1),

$$
F_{i}=\mu N_{i}
$$

which after compensating for mechanical efficiency losses, translates to an equivalent traction limited torque at the motor:

$$
T_{\text {traction, } i}=\left(F_{i}\right) r n / \eta_{m}
$$

The torque available at each motor for acceleration is the minimum of the traction limited torque and the motor limited torque (as defined in the manufacturer's datasheet) at that speed:

$$
T_{i}=\min \left(T_{\text {traction }, i}, T_{\text {motor }, i}\right)
$$

Resistive forces acting on the vehicle due to rolling resistance and drag were calculated using Eq. 4 and 5 respectively.

$$
\begin{gathered}
F_{R R}=C_{r} \sum_{i=1}^{4} N_{i} \\
F_{\text {drag }}=0.5 \rho C_{d} A v_{x}^{2}
\end{gathered}
$$

Similarly, the downforce on the vehicle (assumed to be distributed evenly between each tire for this study) is given by Eq. 6 . A negative lift produces a positive downward force, hence the negative sign at the beginning of the equation.

$$
F_{\text {downforce }}=-0.5 \rho C_{l} A v_{x}^{2}
$$

The final loss modelled under acceleration is due to the rotational inertia of the rotating components in the drivetrain. These can be represented as an equivalent mass within the translational form of Newton's $2^{\text {nd }}$ Law, using the method outlined in [8], which in the reference frame of the vehicle acceleration becomes:

$$
\begin{aligned}
& \frac{\sum_{i=1}^{4} T_{i} n \eta_{m}}{r}-F_{R R}-F_{\text {drag }}=\cdots \\
& =\left(\left(\frac{\sum_{i=1}^{4} I_{\text {Upstream }, i} \cdot n^{2}+\sum_{i=1}^{4} I_{\text {Downstream }, i}}{r^{2}}\right)+m\right) a_{x}
\end{aligned}
$$

The rotational inertia of each drivetrain component was divided into upstream and downstream of the gear ratio to account the increase in apparent inertia of upstream components. Using Eq. 7, the 
longitudinal vehicle acceleration can be determined from the applied motor torques.

The power required from the battery is given by Eq. 8, where the electrical efficiency represents the efficiency of the motor, inverters, cables and battery.

$$
P=\sum_{i=1}^{4} T_{i} \omega / \eta_{e}
$$

When braking, power is recovered through regenerative braking within the motors. Additional braking can be provided by friction brakes if the demanded braking torque exceeds the motor limits.

The rotational components and the vehicle/driver mass were modelled using Simscape components, which are a library of Simulink blocks used for modelling physical systems. The use of these reduces Eq. 7 to a series of connected blocks, with each one representing a physical component such as a motor, gearbox, mass or rotational inertia. Part of the front right drivetrain is shown in Figure 1. This makes it much simpler to adapt the model to potential future changes to the drivetrain layout by changing the order of or replacing components while Simscape deals with the underlying mathematics.

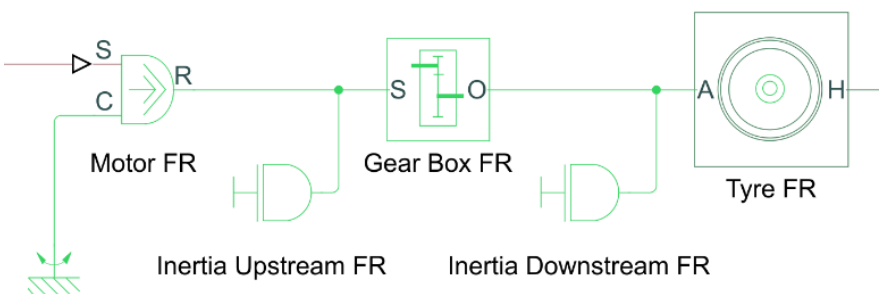

Figure 1. Example drivetrain model using Simscape components.

As the normal force is directly proportional to the tire traction force, the normal force at each tire under longitudinal acceleration considering load transfer and downforce is given by Eq. 9 and 10 . These are derived through balancing moments caused by the reaction force at the front and rear wheels with the vehicle weight and inertial force due to acceleration. Again, note that for this study the downforce is assumed to be distributed evenly between each tire.

$$
\begin{gathered}
N_{F R}=N_{F L}=\frac{m g L\left(1-W_{r}\right)-m a_{x} h}{2 L}+\frac{F_{\text {downforce }}}{4} \\
N_{R R}=N_{R L}=\frac{m g W_{r} L+m a_{x} h}{2 L}+\frac{F_{\text {downforce }}}{4}
\end{gathered}
$$

If the total tire force, $\mathrm{F}_{\mathrm{i}}$, is equal to the Pythagorean sum of the longitudinal and lateral tire forces, then the total remaining vehicle tire force after the application of longitudinal acceleration is given by Eq. 11:

$$
F_{y}=\sum_{i=1}^{4} \sqrt{F_{i}^{2}-F_{x, i}^{2}}
$$

Making the simplifying assumption that the total remaining tire force after longitudinal acceleration can contribute towards cornering (as is the assumption within single tire point mass models such as [9]), a lateral acceleration of a point mass could be achieved, where:

$$
a_{y}=F_{y} / m
$$

Thus for every longitudinal acceleration the model calculates, the corresponding lateral acceleration a point mass could achieve if constantly operating on the tractive limit is derived. There is now a longitudinal acceleration determined considering load transfer and therefore individual traction at each tire, as well as a corresponding lateral acceleration which can be used together to carry out a point mass lap simulation. The lateral acceleration is fed back into the longitudinal model and lateral load transfer is calculated using analogous methodology to Eq 9 and 10. This enables traction shifts in the lateral direction to also be considered when calculating the torque distribution between the motors. To prevent algebraic loops, the model references the accelerations from the previous timestep. Thus the timestep is kept small $(<=0.01 \mathrm{~s})$ to keep the differences negligible.

A MATLAB script programmatically controls the Simulink vehicle model to operate under conditions of acceleration and braking, first at a maximum rate and then at decreasing levels of magnitude. A series of points are generated representing the limit of the vehicle under combined longitudinal and lateral accelerations at velocities from zero to maximum, an example of which is shown in Figure 2. The $x$ and $y$ axes represent the lateral and longitudinal accelerations of the vehicle respectively, whilst the $\mathrm{z}$ axis represents the instantaneous forward velocity. In order to capture the boundary cases, a coastdown from maximum velocity (deceleration only from resistive forces on the vehicle) is also simulated. As there is no longitudinal tire force for acceleration in this case, this represents the maximum possible lateral accelerations, albeit with the vehicle decelerating longitudinally. Similarly, the steady state cornering limits are determined by setting the longitudinal tire force to the value required to counter the deceleration in the coastdown simulation. This represents the boundary of the maximum lateral accelerations possible whilst maintaining a steady velocity.

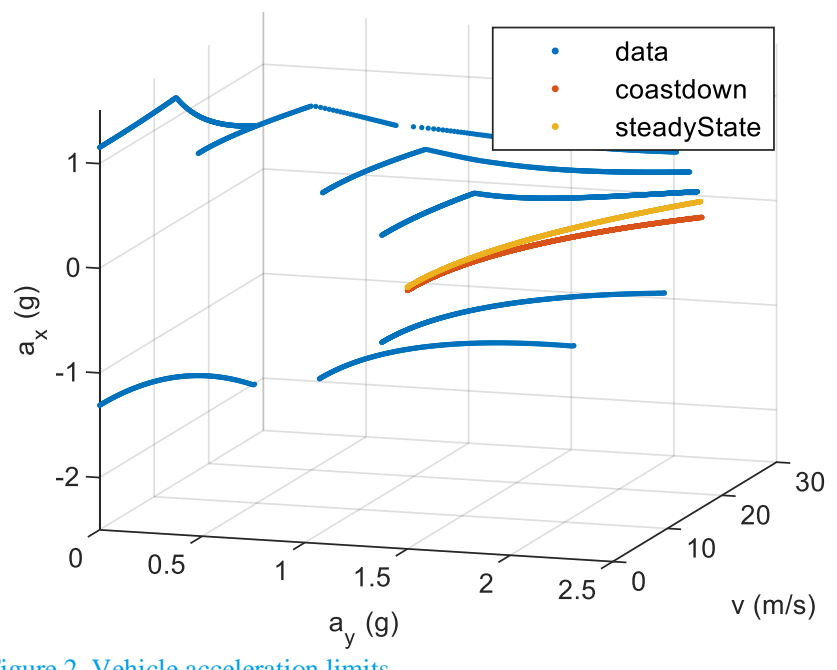

Figure 2. Vehicle acceleration limits.

The data from Figure 2 is split into positive and negative longitudinal accelerations, and a scattered interpolant function used to generate a surface around the points for both acceleration and braking. These surfaces are the ggv diagrams - giving the maximum possible combination of acceleration/braking for a given lateral acceleration and velocity. Examples are shown in Figures 3 and 4, with the same axes representation as Figure 2. The lower edge of the acceleration ggv diagram shows the steady state cornering boundary, whilst the 
upper edge of the braking ggv diagram shows the coastdown deceleration boundary. The same plots can be created for any chosen parameter, such as battery power or motor torques. Thus for any given vehicle acceleration and velocity state the corresponding value of the chosen parameter is also known.

Sampling the data points from these plots during the lap simulation enables the performance to represent a perfect driver driving at the limit of the vehicle, whilst taking into account the limitations in longitudinal acceleration as a result of load transfer and traction shifts. This method also means that the script used to perform the lap simulation only requires the ggv diagrams, and is therefore independent to the vehicle model within Simulink. Changes to the model or vehicle setup do not require any changes in the MATLAB code which controls the lap simulation.

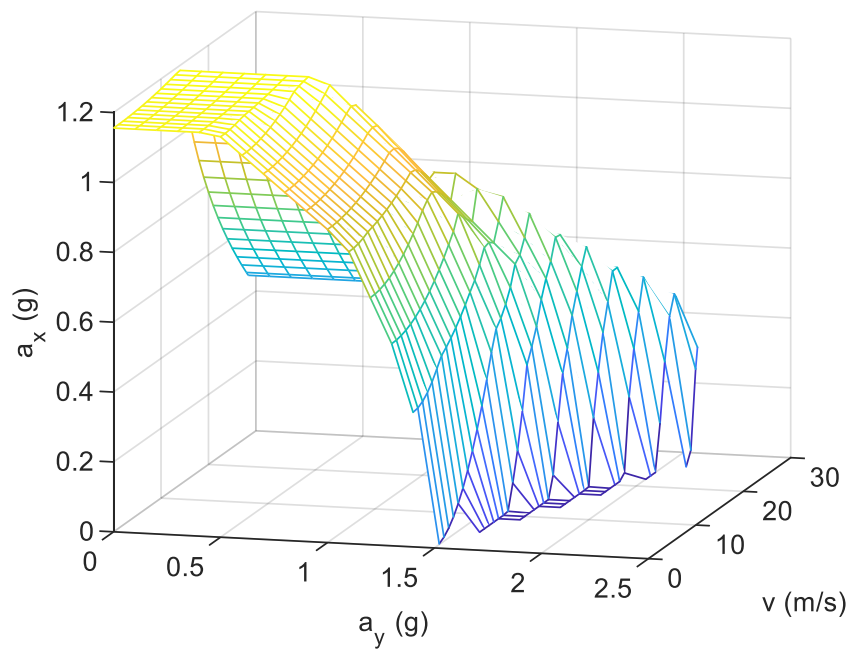

Figure 3. Acceleration ggv diagram.

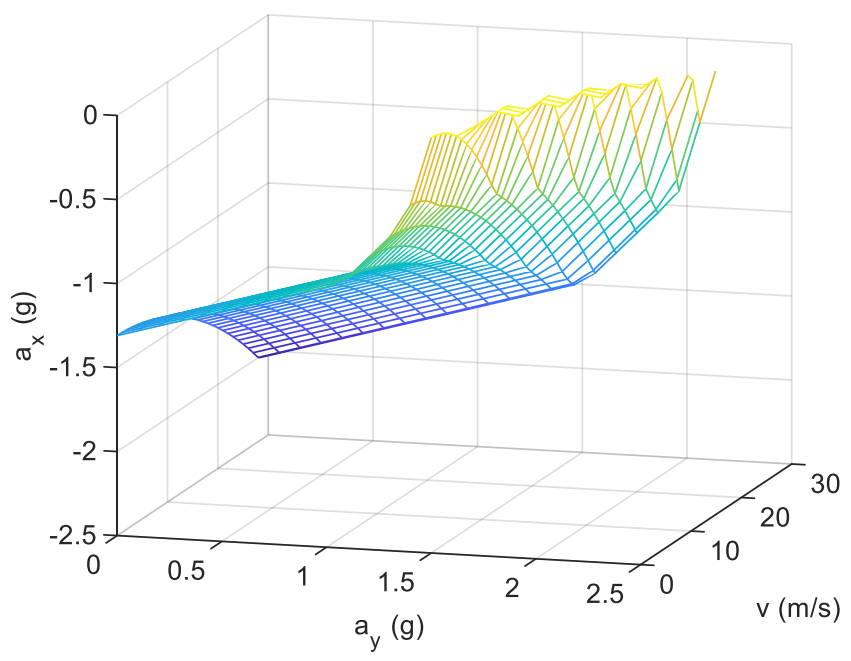

Figure 4. Braking ggv diagram.

\section{Lap Time Simulation}

The tracks used for LTS are split into small equidistant sections. The local curvature, $\mathrm{k}$, is defined for each section, where:

$$
k=\frac{1}{R}
$$

The maximum obtainable corner velocity is set for each section as the steady state cornering limits previously determined. To maintain a curved path, the lateral acceleration is equal to the centripetal acceleration in Eq. 13.

$$
a_{y}=\frac{v_{x}^{2}}{\mathrm{R}}=v_{x}^{2} k
$$

Using the limit of the acceleration ggv diagram where $\mathrm{a}_{\mathrm{x}}=0$, the achievable steady state lateral accelerations are found at every velocity. By rearranging Eq. 13 the maximum curvature possible at each velocity under steady state cornering is obtained. Thus, given the local curvature at each track section, a maximum steady state cornering velocity is set.

Beginning from the first track section, the acceleration ggv diagram is used to determine the achievable velocities under acceleration. The velocity entering each section determines the lateral acceleration required to maintain the local curvature using Eq 13. Using the acceleration ggv diagram as a 2-D lookup table gives the longitudinal acceleration that can be achieved with the instantaneous velocity and lateral acceleration. Thus quasi-static, combined acceleration and cornering is achieved.

Using the equations of motion, the velocity at the end of the track section is given by Eq 14 .

$$
v_{x, j+1}=v_{x, j}+2 a_{x, j}\left(s_{j+1}-s_{j}\right)
$$

If the velocity at a section exceeds the steady state cornering velocity limit, then it is reset to the cornering velocity and acceleration begins again in the subsequent sections. This creates the acceleration velocity profile which indicates the maximum velocity possible at each section based on the car acceleration limits and maximum cornering velocities.

However this does not account for the distance required to decelerate the car to the cornering velocities. By using the same method as before, but instead starting at the final section of the track and working in reverse by using the decelerations from the braking ggv diagram, a braking velocity profile is also created.

By taking the minimum of the acceleration and braking velocity profiles, the final velocity profile across the lap is generating which obeys the limits of the vehicle under acceleration, braking and cornering. This is the same as the method used by OptimumLap [5], with the difference that the accelerations are determined using the ggv method. Figure 5 shows an example of this at the beginning of a lap. The acceleration velocity profile can be seen to increase at the limits of the vehicle until the cornering velocity limit is reached, after which it follows the cornering limit. When the track curvature decreases causing the corner velocity limit to increase, the acceleration velocity profile increases again at the limit of the vehicle. Similarly, moving across the plot from right to left, the braking velocity profile follows the corner speed limit until the track curvature decreases, allowing it to increase at a magnitude equal to the maximum rate of braking. The intersections between the two velocity profiles show the transitions from acceleration to braking and vice versa. 
Finally, using the combined velocity profile, the combined longitudinal acceleration and overall time at each section are calculated using Eq. 15 and 16.

$$
\begin{gathered}
a_{x, j}=\frac{v_{x, j+1}{ }^{2}-v_{x, j}^{2}}{2\left(s_{j+1}-s_{j}\right)} \\
t_{j+1}=t_{j}+\frac{2\left(s_{j+1}-s_{j}\right)}{v_{x, j}+v_{x, j+1}}
\end{gathered}
$$

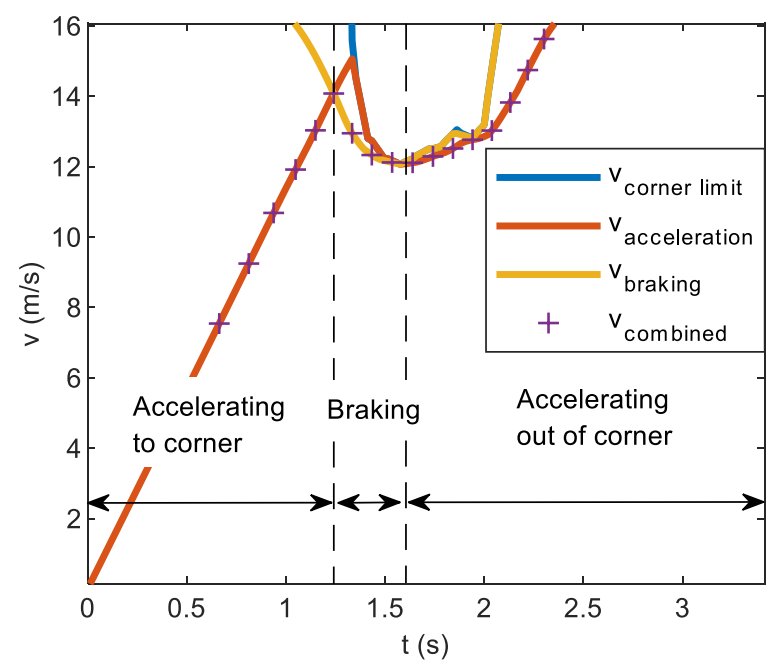

Figure 5. Combined acceleration/braking and cornering.

\section{Limitations}

Any simulation is an abstraction of reality based on the assumptions made. Knowledge of the following limitations are important in assigning validity to the results:

1. The linear tire model used does not account for wheel slip, toe, camber, temperature or pressure. Total tire force was assumed to be the Pythagorean sum of the lateral and longitudinal components.

2. The point mass LTS method neglects yaw motion.

3. Transient effects of suspension and multibody dynamics were ignored.

4. The quality of the track data used in the model can influence the simulated time. Depending on the method of capturing the track, (eg onboard GPS), noise in the measured values can cause unrepresentative changes in local track curvature.

5. A perfect driver is modelled - thus the LTS is expected to produce times quicker than reality.

Nevertheless, the tool acts as a useful comparative analysis between different vehicle concepts, without the inconsistencies of a driver.

\section{Results}

\section{Comparison to OptimumLap}

Predicted lap times were compared for three FS tracks between OptimumLap and the LTS tool in Table 1. As expected, the LTS times were slower than OptimumLap $(1.18 \%-1.74 \%)$ due to the inclusion of rotational inertia and load transfer. The difference was not as significant as expected - possibly due to the four proposed 38 $\mathrm{kW}$ motors having enough surplus power to cope with load transfer as well as the maximum power limit imposed in the rules keeping the tires far below their tractive limits. To evaluate this, the power at each motor was scaled down to $65 \%$, and the power draw limit removed. The comparison was repeated, with the results shown in Table 2. The difference between OptimumLap and the LTS tool increased in this case to $1.91 \%-2.28 \%$.

Thus it is likely the LTS tool offers greater advantages over OptimumLap when used to simulate lap times in vehicles other than those competing in FS. Nevertheless, a difference of $1.74 \%$ could be considered significant in racing, with the difference between $1^{\text {st }}$ and $2^{\text {nd }}$ place in the endurance event at the three competitions listed being $3.7 \%, 2.8 \%$ and $0.75 \%$ respectively. In addition, the methodology used in this LTS tool has other benefits:

1. Parameters are simulated at each of the four drivetrains independently - potentially useful for concept development (for example creating torque duty cycles for component sizing in each drivetrain).

2. The effects of changing the vehicle center of mass can be evaluated.

3. Changes to the model can be implemented using Simscape's graphical interface whilst the LTS code remains unchanged.

4. Extra features not available in OptimumLap can be easily built in such as drag reduction systems or detailed motor efficiency maps when more data is available.

Table 1. Comparison between OptimumLap and LTS predicted lap times for three FS tracks.

\begin{tabular}{|l|l|l|l|}
\hline & Austria 2012 & Lincoln 2012 & Michigan 2014 \\
\hline OptimumLap & $51.77 \mathrm{~s}$ & $65.6 \mathrm{~s}$ & $60.02 \mathrm{~s}$ \\
\hline LTS & $52.67 \mathrm{~s}$ & $66.38 \mathrm{~s}$ & $60.80 \mathrm{~s}$ \\
\hline Difference (\%) & $1.74 \%$ & $1.18 \%$ & $1.31 \%$ \\
\hline
\end{tabular}

Table 2. Comparison between OptimumLap and LTS predicted lap times for three FS tracks with $65 \%$ of motor power and no battery power draw limit.

\begin{tabular}{|l|l|l|l|}
\hline & Austria 2012 & Lincoln 2012 & Michigan 2014 \\
\hline OptimumLap & $51.68 \mathrm{~s}$ & $65.43 \mathrm{~s}$ & $59.88 \mathrm{~s}$ \\
\hline LTS & $52.86 \mathrm{~s}$ & $66.73 \mathrm{~s}$ & $61.03 \mathrm{~s}$ \\
\hline Difference (\%) & $2.28 \%$ & $1.98 \%$ & $1.91 \%$ \\
\hline
\end{tabular}

\section{Validation}

With the QUB EV only a concept at this stage, validation of the LTS tool with test data was not possible. However, Formula Student Team Delft publish limited vehicle specifications on their website, including details of their vehicle entered in to Formula Student Germany (FSG) in 2012, known as DUT 12. Vehicle information is 
available at [10]. DUT 12 was powered by motors of the same torque output and manufacturer as those proposed by QUB, and had no aero package making it a similar concept. QUB have access to the FSG 2012 endurance track data, which combined with the DUT 12 vehicle parameters provides the inputs required for simulation within the LTS tool. Comparing the simulated performance with the published competition results from that year provides a useful source of preliminary validation of the LTS tool, as shown in Table 3.

Table 3. Comparison between simulated and actual results.

\begin{tabular}{|l|l|l|}
\hline & Acceleration Event & Endurance Event \\
\hline Competition Time & $3.454 \mathrm{~s}$ & $78.297 \mathrm{~s}$ \\
\hline Simulated Time & $3.494 \mathrm{~s}$ & $75.234 \mathrm{~s}$ \\
\hline Difference (\%) & $1.17 \%$ & $-3.91 \%$ \\
\hline
\end{tabular}

The predicted endurance lap time is quicker than reality, despite the simulated straight line performance being slightly slower. This means that the error is coming from the curved sections, which is intuitive given the assumptions of a perfect driver and point mass cornering. It is worth noting that the predictions are subject to the assumptions made in the model methodology as well as estimation of some unpublished DUT 12 vehicle parameters. Although more examples are needed to validate the tool, the accuracy in this situation is well within a useful range for early concept evaluation and development. With the assumptions made, the LTS tool was not intended to be used for predicting the lap times of a particular vehicle with absolute precision - the value of the tool is as a comparative analysis between different vehicles as a rapid concept evaluation.

\section{Powertrain Specification}

To optimize the powertrain gear ratio and battery capacity, it was assumed that the proposed four in-hub motor concept would be used, with the same tires and chassis (with no aero package) to previous QUB ICE vehicles [11]. Thus the same geometry and coefficients could be used. Mass was estimated for an EV based on the substituted components from the ICE car, rotational inertia of drivetrain components estimated from CAD models.

\section{Transmission Gear Ratio}

Figure 6 shows the simulated change in acceleration event and endurance lap times for a range of gear ratios. A gear ratio of 14 minimizes the $75 \mathrm{~m}$ acceleration event time, whilst 11 produces the quickest endurance lap time. However, the difference in simulated times for each event between gear ratios of 11 and 14 is small compared to the spread of times encountered in the competition (1.05 $\%$ and $0.328 \%$ compared to $150 \%$ and $145 \%$ ). In FSUK 2018, neither of the first place teams in each event would have lost any points if the same percentage difference was applied to their times. Therefore it is likely that any gear ratio in the range of $11-14$ would be acceptable.

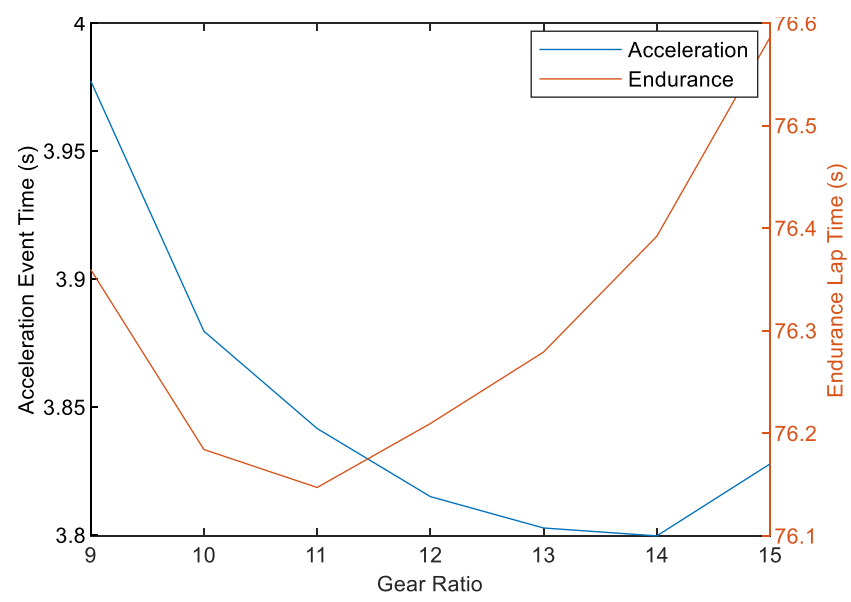

Figure 6. Influence of gear ratio on acceleration event and endurance lap time.

To understand why the optimum gear ratio is different for each event, Figure 7 was plotted which shows the longitudinal acceleration performance against distance into a straight section for each gear ratio. The higher gear ratio offers better acceleration off the line, however over the course of a $22 \mathrm{~km}$ endurance event, the vehicle only accelerates from a standstill twice. The higher gear ratio also requires the motor to spin faster for the same vehicle velocity, thus the $80 \mathrm{~kW}$ power draw limit is reached sooner and the asymptotic torque decrease begins earlier. Most significantly, the higher gear ratio reaches run out speed $65.2 \mathrm{~m}$ after accelerating from a standstill. With the maximum straight in an endurance track being up to $80 \mathrm{~m}$ [7], combined with the vehicle entering the straights already at speed, the benefit of the increased maximum velocity is more significant than off the line performance in the endurance event.

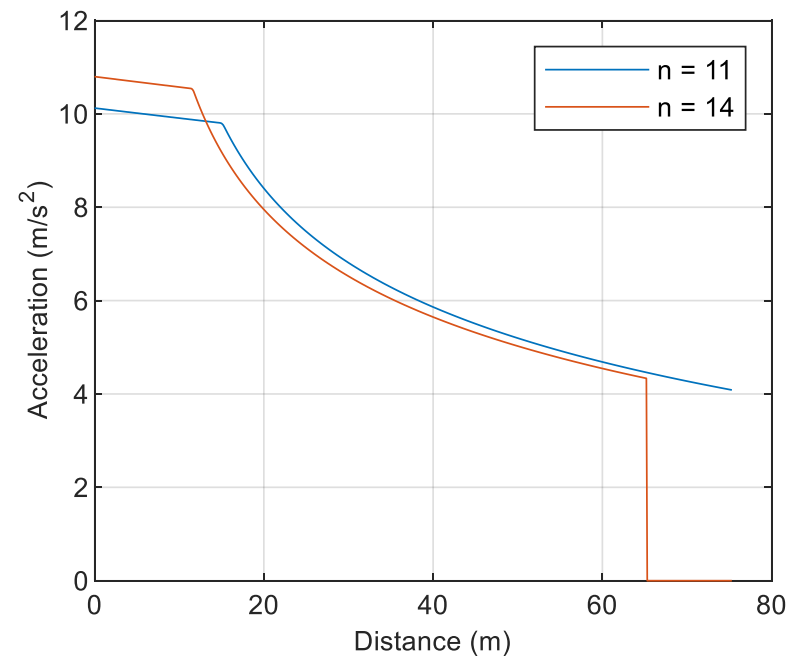

Figure 7. Longitudinal acceleration performance for each gear ratio.

Figure 8 plots the difference in velocity (delta v) between a gear ratio of 11 and 14 across a bird's eye view of a flying start endurance lap to confirm this - the difference is negligible apart from when reaching run out speed on a longer straight section. It is possible that in reality, a non-perfect driver would decrease the average velocity and prevent run out speed, reducing this effect.

However with the simulated difference between the setups so small, it is recommended to choose a gear ratio of 11 for several reasons: 
1. Preventing running the motors at maximum speed should prolong their life, decrease temperatures and operate at a higher efficiency point.

2. Less mechanical drivetrain losses as heat.

3. Future development will aim to decrease the vehicle mass. This will decrease the normal force and thus traction available for off the line performance, as well as requiring less force to accelerate the car. Thus gearing up the torque will become less necessary.

4. FS has two events involving laps of a track - the endurance and the sprint events. Both are worth more competition points than the acceleration event.

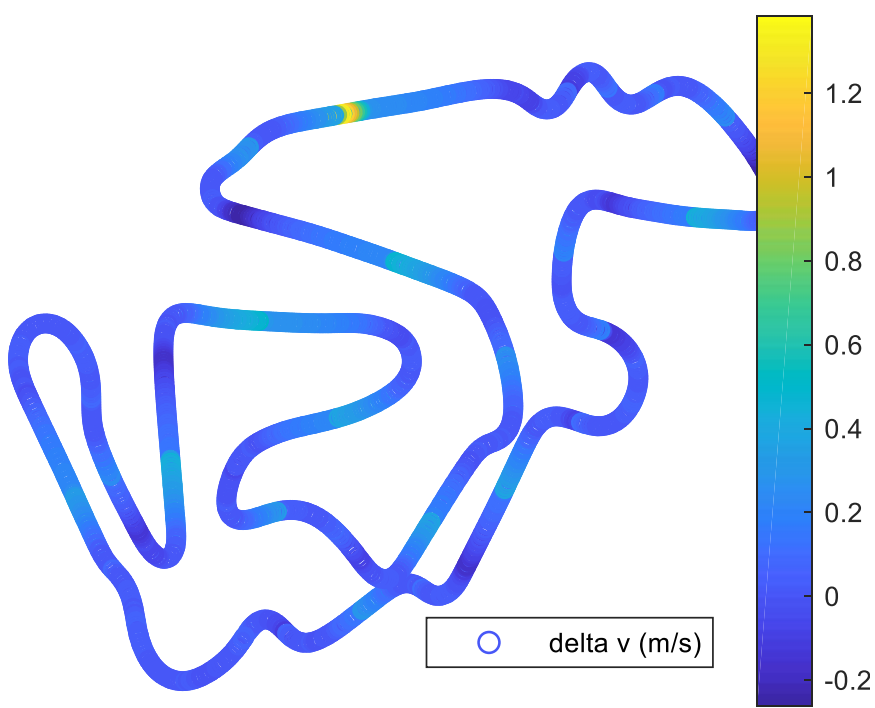

Figure 8. Difference in velocity between a gear ratio of 11 and 14 across a bird's eye view of a flying start endurance lap.

\section{Battery Pack Capacity}

Using a gear ratio of 11, the FSG 2012 endurance event was simulated. Battery power from regenerative braking was capped at 45 $\mathrm{kW}$ as per [12]. The endurance event in 2012 featured 18 laps at 1.22 $\mathrm{km}$ each, completed in two sets of nine laps. This means that two laps began from a standstill, whilst the remaining 16 had a flying start. Figure 9 shows the simulated battery power draw for a flying start lap, with negative power indicating regenerative braking.

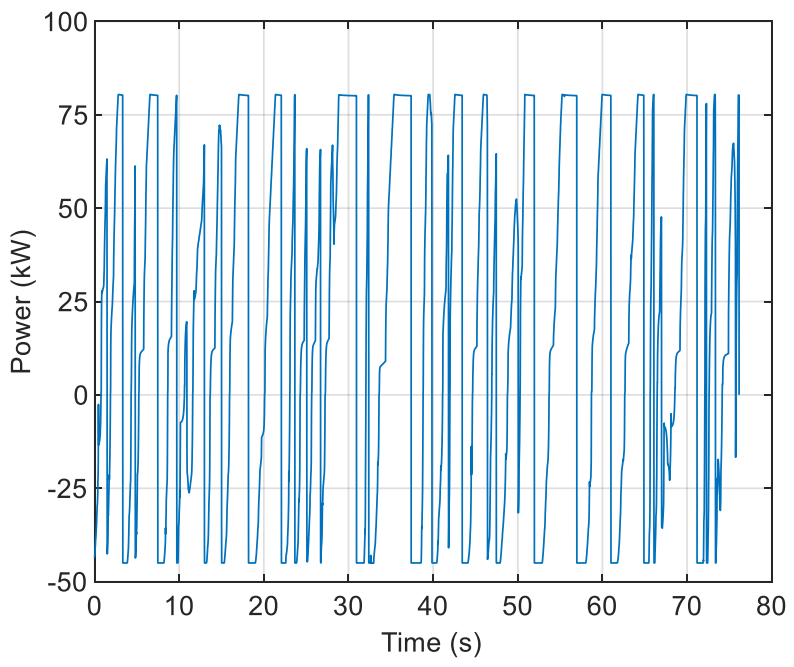

Figure 9. Battery power draw across a flying start endurance lap.
Table 4 displays the total energy required for each lap, along with the energy recovered through regenerative braking. Across the complete $22 \mathrm{~km}$ endurance event, a battery of $6.764 \mathrm{kWh}$ capacity is required to complete the event under maximum performance. This is the limiting consideration on battery capacity, as it is the longest event and the battery can be fully charged prior to starting.

Table 4. Energy usage across a $22 \mathrm{~km}$ endurance event.

\begin{tabular}{|l|l|l|l|}
\hline & $\begin{array}{l}\text { Standing Start } \\
\text { Lap }\end{array}$ & $\begin{array}{l}\text { Flying Start } \\
\text { Lap }\end{array}$ & $\begin{array}{l}\text { Endurance } \\
\text { Event Total }\end{array}$ \\
\hline $\begin{array}{l}\text { Energy Used } \\
(\mathrm{kWh})\end{array}$ & 0.6667 & 0.6610 & 11.909 \\
\hline $\begin{array}{l}\text { Regen Energy } \\
\text { (kWh) }\end{array}$ & 0.2821 & 0.2863 & 5.145 \\
\hline $\begin{array}{l}\text { Energy } \\
\text { Required (kWh) }\end{array}$ & 0.3846 & 0.3747 & 6.764 \\
\hline
\end{tabular}

It is worth noting that the mass of the vehicle for these simulations was estimated by QUB, which includes an estimated battery mass. It is likely that the actual mass of the vehicle and battery will differ, and thus the required energy for the completion of the endurance event will differ. As battery capacity is directly related to battery mass, changing the battery capacity to reflect the new energy requirement would again change the battery mass - subsequently creating another updated energy requirement. Thus an iterative process is required to converge on an optimum battery capacity. This should be performed pending the availability of more accurate vehicle mass predictions.

\section{Sensitivity Analysis}

Variation of acceleration event and endurance lap times in response to changes in eight parameters were investigated to determine those most critical to performance. Frontal area, drag coefficient and lift coefficient were chosen as they are key factors in aerodynamic performance and there is significant room for development. Similarly, the vehicle mass and center of mass position are parameters QUB can change in subsequent years, and are key factors in acceleration and load transfer. Electrical efficiency, rolling resistance coefficient and tire friction coefficient are included to understand the sensitivity of the model to estimated parameters and thus the need to obtain more accurate values.

500 cases were generated by varying the eight parameters within individual ranges which were deemed as achievable through future vehicle development. A Latin Hypercube sampling method was used to set the combinations of parameters for each case. The Latin Hypercube sampling technique stratifies the input parameters of a multivariate problem, to avoid clustering of random samples and ensure every portion of each parameter is represented with a sample [13]. Each case was then simulated, and a response surface fitted to the results, where the local gradient for each parameter shows the sensitivity of the predicted times in response to each design variable.

Figures 10 and 11 show the sensitivity analyses for the acceleration and endurance events respectively. The steeper the gradient in each plot, the more sensitive the predicted time is within the specified parameter design space. For the acceleration event, the mass, 
electrical efficiency and drag coefficient are significant parameters. Targeting weight reduction in future development is critical in reducing the acceleration event time. However the drag coefficient is already on the lower side of the achievable design space, but the addition of an aero package (which is a future goal of QUB) to the car would cause it to increase leading to a trade-off consideration. To add to this the mass and frontal area would also increase, negatively impacting the time further. The electrical efficiency has a large influence on the acceleration time, which in reality is likely a constraint of the electrical components selected and therefore difficult to change. Its significance to this tool however is that it is an estimated value, which therefore must be substituted with accurate data when it becomes available.

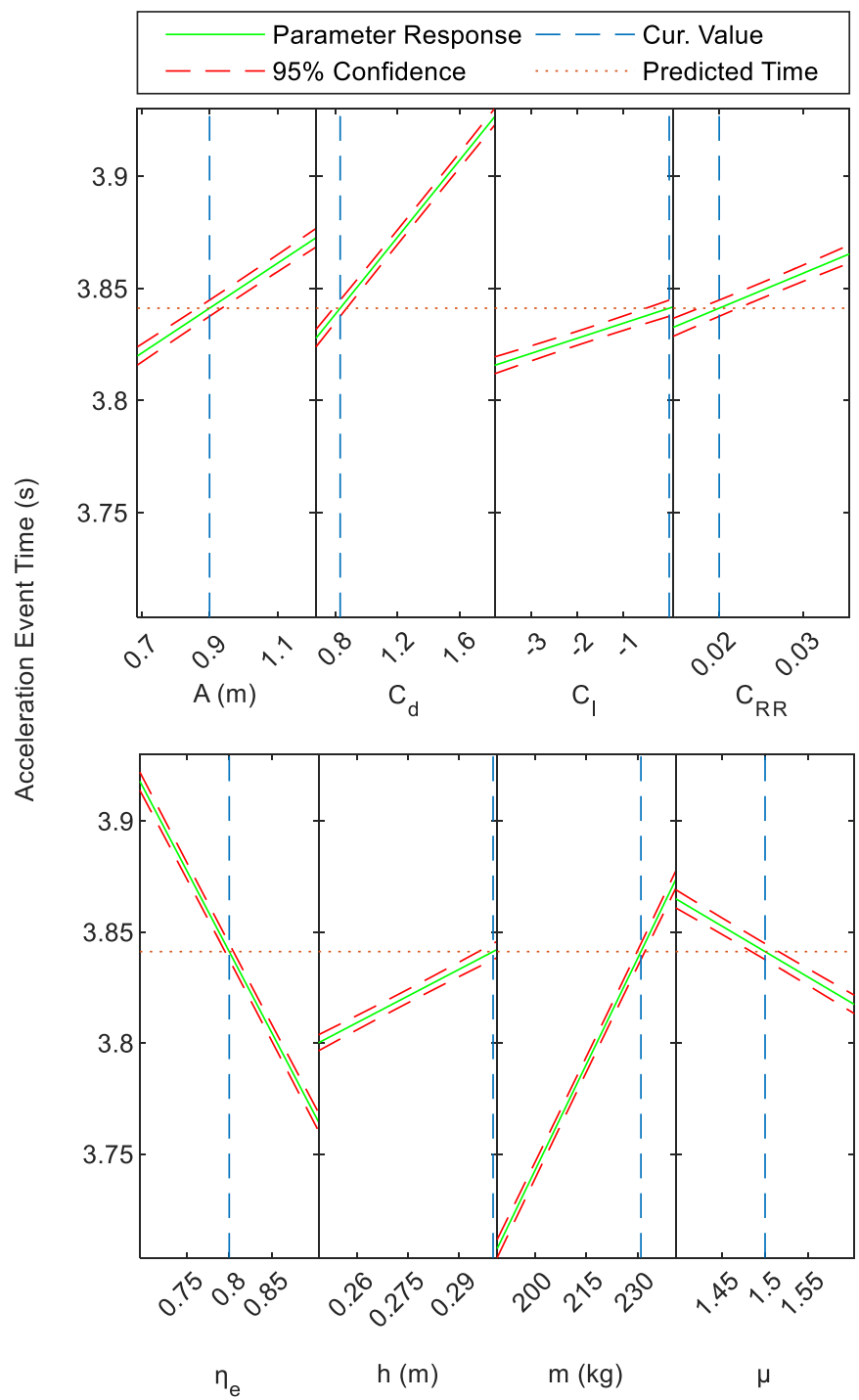

Figure 10. Acceleration event sensitivity analysis.

The endurance time is particularly sensitive to the lift coefficient. In this case the benefits of the extra downforce are so significant that the simultaneous increase in frontal area is actually beneficial (assuming that the extra area is generating downforce), whilst the increase in drag is not as limiting as the acceleration event. Although decreasing the lift coefficient in isolation also slightly decreases the acceleration event time, increases in mass, drag and area are more significant within the design space. Further analysis is required to justify an aero package, with a consideration of competition points lost and gained between the acceleration and endurance events using simulated times.

The tire friction coefficient is a significant factor in both events, and in particular the endurance lap time. This highlights the limitations of the simplified tire model used. It can also be used as an insight into how different road surfaces or conditions may affect the lap time, and allow a comparison between slick and wet tires.

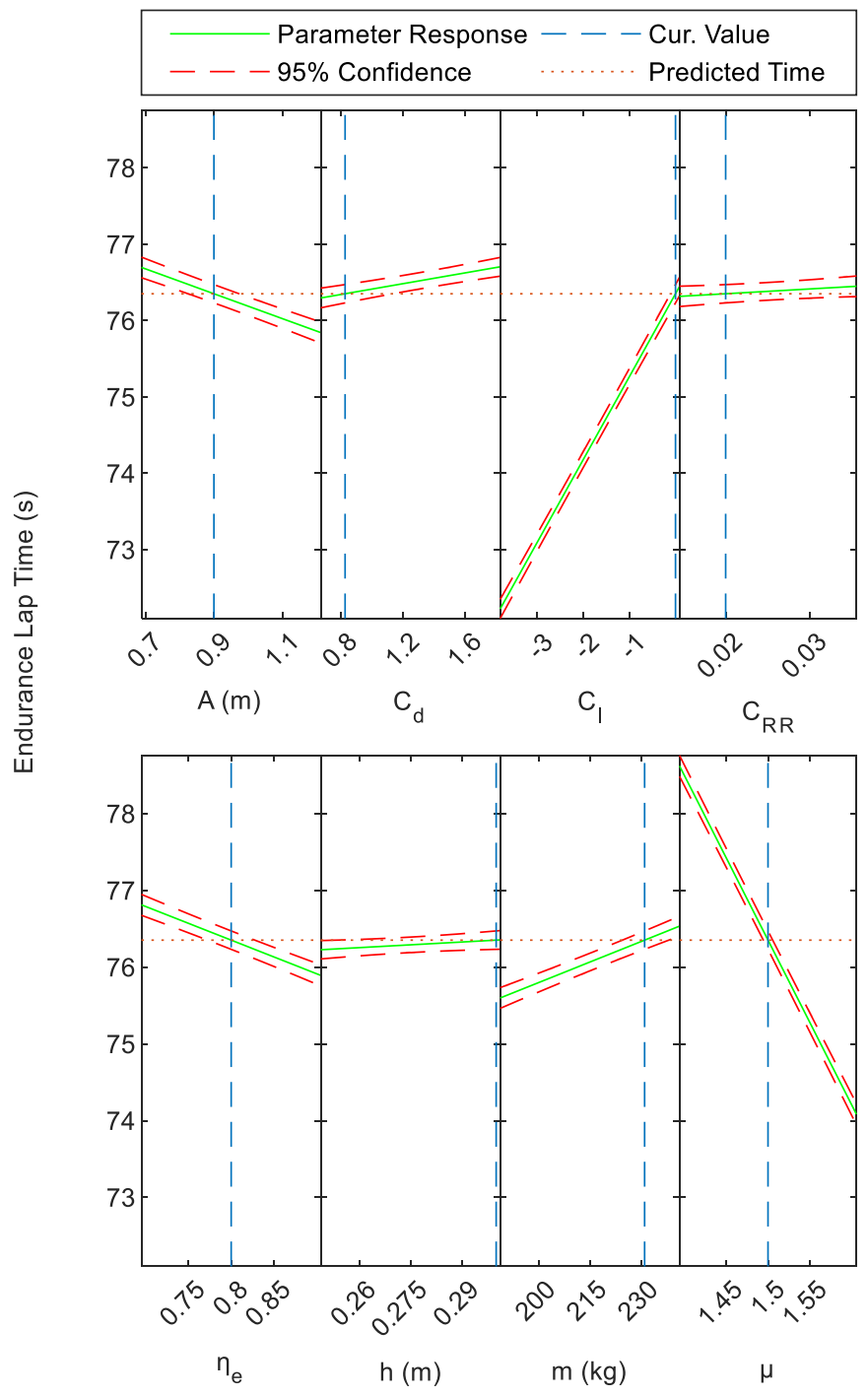

Figure 11. Endurance lap sensitivity analysis.

Assigning a cost per unit change to each of the design parameters allows a cost weighting to be allocated to a unit increase in performance based on each parameter. With a small budget and limited resource, this can show the most economical areas to spend funding and therefore influence future development.

\section{Conclusions}

A point mass, quasi-static LTS tool capable of combined cornering and accelerating/braking has been developed. A four-wheeled vehicle model was created using Simulink which generates the performance envelope of the vehicle in the form of a ggv diagram - which is then used to perform the lap simulation. This offers a method of 
accounting for traction shifts as a result of load transfer within a point mass LTS.

The new method enables the simulation of parameters individual to each of the four drivetrains, which gives more insight to a team developing a FS car in informing design decisions. It also separates the vehicle model from the MATLAB code required to run the LTS, reducing the complexity of implementing future changes. The use of Simscape to represent physical drivetrain components offers a graphical interface, with the underlying mathematics automatically generated. Thus, the LTS aims to reduce the impact of knowledge loss between subsequent FS teams through ease of adaptability.

The developed tool was compared to the freeware point mass LTS software, OptimumLap. As expected, the new method predicted slower lap times with a difference in the range of $1.18 \%-1.74 \%$ across three test FS tracks. This result was less significant than previously expected, likely due to the four $38 \mathrm{~kW}$ proposed motors having enough surplus power to cope with traction shifts, and the 80 $\mathrm{kW}$ power limit imposed in FS keeping the vehicle well below the tractive limit at higher speeds. Removing this limit and scaling the motor power by $65 \%$ caused the difference between the LTS and OptimumLap to increase to $1.91 \%-2.28 \%$. In the absence of a test vehicle, validation against DUT 12 at FSG 2012 was attempted, with an error of $1.17 \%$ and $-3.91 \%$ for the quickest acceleration event and endurance lap times.

The single transmission gear ratio for the proposed QUB EV was specified as 11 through consideration of the simulated acceleration event and endurance lap times. The required battery capacity of 6.764 $\mathrm{kWh}$ was determined through simulation of a $22 \mathrm{~km}$ endurance event. A sensitivity analysis was performed to guide future QUB resource allocation. Decreasing vehicle mass, center of gravity height and rolling resistance coefficient would increase performance in both the acceleration and endurance event with no trade-offs to be considered with other parameters. Frontal area, lift and drag coefficients are codependent, with trade-offs both between each other and in optimizing between either the acceleration or endurance events. Further analysis is therefore required to quantify the effects of an aero package.

\section{References}

1. SAE International, "Formula SAE Online," https://www.fsaeonline.com, accessed Oct. 2018.

2. Srinivasan, P. and M, K., "Performance Fuel Economy and CO2 Prediction of a Vehicle using AVL Cruise Simulation Techniques," SAE Technical Paper 2009-01-1862, 2009, https://doi.org/10.4271/2009-01-1862.

3. Govender, V. and Barton, D., "Multibody System Simulation and Optimisation of the Driving Dynamics of a Formula SAE Race Car," SAE Technical Paper 2009-01-0454, 2009, https://doi.org/10.4271/2009-01-0454.

4. Costa, R. and Bortolussi, R., "Lap Time Simulation of Formula SAE Vehicle With Quasi-steady State Model," SAE Technical Paper 2016-36-0164, 2016, https://doi.org/10.4271/2016-360164.

5. OptimumLap (Version 1.4.2), Computer Software, OptimumG, Denver, CO, 2012.

6. Sakhalkar, S., Dhillon, P., Bakshi, S., Kumar, P., and Arora, P.S., "Powertrain Model for Selection of Reduction Ratio and Estimation of Energy Requirement," SAE Technical Paper 2014-01-1781, 2014, https://doi.org/10.4271/2014-01-1781.
7. IMechE, 'Formula Student Rules 2018," https://www.imeche.org/events/formula-student/teaminformation/rules, accessed Oct. 2018.

8. Stevens, G., Murtagh, M., Kee, R., Early, J. et al., "Development of a Vehicle Model Architecture to Improve Modeling Flexibility," SAE Int. J. Engines 10(3):1328-1366, 2017, https://doi.org/10.4271/2017-01-1138.

9. Criens, C., Dam, T. ten, Luijten, H., and Rutjes, T., "Building a MATLAB based Formula Student simulator," Master Team Proj. Report, Department Mechanical Engineering, Technische Universiteit Eindhoven, Eindhoven, 2006.

10. Formula Student Team Delft, "DUT 12," https://www.fsteamdelft.nl/dut12, accessed Jan. 2019.

11. White, G., Cunningham, G., and Doyle, D., "Design of an Electric Drive Transmission for a Formula Student Race Car," SAE Technical Paper (number to be confirmed), 2019.

12. August Aune, P., "A Four Wheel Drive System for a Formula Style Electric Racecar," Master thesis, Department of Product Design, Norwegian University of Science and Technology, Trondheim, 2016.

13. Wang, G.G., "Adaptive Response Surface Method Using Inherited Latin Hypercube Design Points," J. Mech. Des 125(2):210-220, 2003, https://doi.org/10.1115/1.1561044.

\section{Acknowledgements}

The author would like to thank Dr Geoff Cunningham for his frequent time and support throughout this project, along with the QUB EV team members Gavin White and Connor McShane. Thanks also to Dr Juliana Early for her guidance.

\section{Contact Information}

Darryl Doyle

ddoyle08@qub.ac.uk

\section{Nomenclature}

$\mu$

a

$\mathbf{A}$

$\mathbf{C}_{\mathrm{d}}$

$\mathbf{C}_{1}$

$\mathrm{C}_{\mathrm{RR}}$

F

g

h

I

$\mathbf{k}$
Tire friction coefficient

Acceleration (m/s $\left.\mathrm{s}^{2}\right)$

Frontal area $\left(\mathrm{m}^{2}\right)$

Drag coefficient

Lift coefficient

Rolling resistance coefficient

Total tire force $(\mathrm{N})$

Gravitational acceleration $\left(\mathrm{m} / \mathrm{s}^{2}\right)$

Center of mass height (m)

Rotational Inertia (kg.m²)

Local track curvature $(1 / \mathrm{m})$ 
Power (W)

Tire rolling radius $(\mathrm{m})$

Local track radius (m)

Displacement (m)

Time (s)

Motor torque (Nm)

Velocity (m/s)

Rear weight distribution (\%)

Efficiency (\%)

Air density $\left(\mathrm{kg} / \mathrm{m}^{2}\right)$

Motor angular velocity ( $\mathrm{rad} / \mathrm{s})$

\section{Subscripts}

e

i

j

m
Electrical

Motor/tire identication ( $1=\mathrm{FR}$,

$2=\mathrm{FL}, 3=\mathrm{RR}, 4=\mathrm{RL}$ )

Track section

Mechanical motor

RR

traction

$\mathbf{x}$

$\mathbf{y}$

Motor limited

Rolling resistance

Traction limited

Longitudinal direction

Lateral direction

\section{Definitions/Abbreviations}

DUT 12

Delft University of Technology

2012

EV

Electric Vehicle

FL

Front Left

FR

Front right

FS

Formula Student

FSG

Formula Student Germany

FSUK

Formula Student United

Kingdom

ICE

Internal Combustion Engine

LTS

Lap time simulation

QUB

Queen's University Belfast

RL

Real Left

RR

Rear Right 